gap $>\mathrm{g}:=$ SymmetricGroup $(4)$;

$\operatorname{Sym}\left(\left[\begin{array}{lll}1 & 4\end{array}\right]\right)$

gap> tbl:= CharacterTable ( $\mathrm{g}$ ); ; HasIrr ( tbl );

false

05 = total: $\begin{array}{rrrrr}1 & 4 & 13 & 14 & 4\end{array}$

gap> tblmod2:= CharacterTable( tbl, 2 );

BrauerTable( $\operatorname{Sym}([1 \ldots 4$ ] ), 2 )

1: 2242 gap> tblmod2 = CharacterTable $(t b 1,2$ );

2: . 256 . true

Journal of Software for

01234 gap> libtbl:= CharacterTable( "M" );

$06=$ total: $1{ }^{4} 13144$ CharacterTable ( "M")

fail

gap> CharacterTable ( "Symmetric", 4 ); int a, b, c, t=11, 5, 3,0;

06 : BettiTally

CharacterTable ( "Sym(4)" )

gap> ComputedBrauerTables ( tbl );

$\begin{array}{rrrrrr}0 & 1 & 2 & 3 & 4 \\ 1 & 4 & 13 & 14 & 4\end{array}$

[, BrauerTable $(\operatorname{Sym}([1 \ldots 4]), 2$ poly $f=x^{\wedge} a+y^{\wedge} b+z^{\wedge}(3 * c)+x^{\wedge}(c+2) * y^{\wedge}(c-1)+x^{\wedge}$ $\mathrm{x}^{\wedge}(\mathrm{c}-2) * \mathrm{y}^{\wedge} \mathrm{c} *\left(\mathrm{y}^{\wedge} 2+\mathrm{t} * \mathrm{x}\right) \sim 2$;

\begin{tabular}{|c|c|c|c|}
\hline 0: & 1 & & \\
\hline 1: & . & & \\
\hline 2: & . & & \\
\hline 3: & & & \\
\hline 4: & . & & \\
\hline 5: & & & \\
\hline 6: & . & & \\
\hline $7:$ & 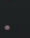 & & \\
\hline & . & & \\
\hline
\end{tabular}

o7 : BettiTally

i8 : peek t1

$08=\operatorname{BettiTally}\{(0,\{0,0\}, 0) \Rightarrow 1\}$

$(1,\{2,2\}, 4) \Rightarrow 2$

$(1,\{3,3\}, 6) \Rightarrow 2$

$(2,\{3,7\}, 10) \Rightarrow 2$

$(2,\{4,4\}, 8) \Rightarrow 1$

$(2,\{4,5\}, 9) \Rightarrow 4$

$(2,\{5,4\}, 9) \Rightarrow 4$

$(2,\{7,3\}, 10) \Rightarrow 2$

$(3,\{4,7\}, 11) \Rightarrow 4$

$(3,\{5,5\}, 10)$ The relative canonical resolution:

(3, 4 acautay $\Rightarrow 2$ package, experiments and conjectures

$(4,\{7,5\}, 12) \Rightarrow 2$

Christian BopP AND Michael HofF 


\title{
The relative canonical resolution: Macaulay2-package, experiments and conjectures
}

\author{
Christian BopP AND Michael HoFF
}

\begin{abstract}
This short note provides a quick introduction to relative canonical resolutions of curves on rational normal scrolls. We present our Macaulay 2 package that computes the relative canonical resolution associated to a curve and a pencil of divisors. We end with a list of conjectural shapes of relative canonical resolutions. In particular, for curves of genus $g=n \cdot k+1$ and pencils of degree $k$ for $n \geq 1$, we conjecture that the syzygy divisors on the Hurwitz scheme $\mathscr{H}_{g, k}$ constructed by Deopurkar and Patel (Contemp. Math. 703 (2018) 209-222) all have the same support.
\end{abstract}

\section{Relative CANONiCAl Resolutions.}

The relative canonical resolution is the minimal free resolution of a canonically embedded curve $C$ inside a rational normal scroll. Every such scroll is swept out by linear spaces parametrized by pencils of divisors on $C$.

Studying divisors on moduli spaces reveals certain aspects of the global geometry of these spaces. A famous example for odd genus $g$ is the Koszul divisor on the moduli space of curves $\mathscr{M}_{g}$ (see [Hirschowitz and Ramanan 1998; Voisin 2005; Farkas 2009]). It can be derived from the minimal free resolution of $C \subset \mathbb{P}^{g-1}$. Set-theoretically the Koszul divisor consists of curves such that the minimal free resolution of the canonical model has extra syzygies at a certain step. In [Bujokas and Patel 2015; Deopurkar and Patel 2015; 2018], the relative canonical resolution was used to define similar syzygy divisors on Hurwitz spaces $\mathscr{H}_{g, k}$, parametrizing pairs of curves of genus $g$ and pencils of divisors of degree $k$ (equivalently, covers of $\mathbb{P}^{1}$ of degree $k$ by curves of genus $g$ ). We also refer to [Farkas 2018] for divisors on Hurwitz spaces.

We will briefly summarize the connection between pencils of divisors on canonical curves and rational normal scrolls in order to define the relative canonical resolution. The following definition and statements can be found in [Harris 1981, §3] and [Schreyer 1986, §1]. Let $C \subset \mathbb{P}^{g-1}$ be a canonically embedded curve of genus $g$, and let

$$
g_{k}^{1}=\left\{D_{\lambda}\right\}_{\lambda \in \mathbb{P}^{1}} \subset|D|
$$

MSC2010: 13D02, 14H51, 14Q05.

Keywords: Hurwitz space, syzygy modules, relative canonical resolution.

RelativeCanonicalResolution version 1.0 
be a pencil of divisors of degree $k$. If we denote by $\overline{D_{\lambda}} \subset \mathbb{P}^{g-1}$ the linear span of the divisor, then

$$
X:=\bigcup_{\lambda \in \mathbb{P}^{1}} \overline{D_{\lambda}} \subset \mathbb{P}^{g-1}
$$

is a $(k-1)$-dimensional rational normal scroll of degree $\operatorname{deg}(X):=f=g-k+1$.

Definition 1.1. Let $e_{1} \geq e_{2} \geq \cdots \geq e_{d} \geq 0$ be integers, $\mathscr{E}=\mathscr{O}_{\mathbb{P}^{1}}\left(e_{1}\right) \oplus \cdots \oplus \mathscr{O}_{\mathbb{P}^{1}}\left(e_{d}\right)$, and let $\pi: \mathbb{P}(\mathscr{E}) \rightarrow \mathbb{P}^{1}$ be the corresponding $\mathbb{P}^{d-1}$-bundle. A rational normal scroll of type $\left(e_{1}, \ldots, e_{d}\right)$ is the image of

$$
j: \mathbb{P}(\mathscr{E}) \rightarrow \mathbb{P} H^{0}\left(\mathbb{P}(\mathscr{E}), \mathscr{O}_{\mathbb{P}(\mathscr{E})}(1)\right)=\mathbb{P}^{r} .
$$

Note that $r=f+d-1$ with $f=e_{1}+\cdots+e_{d} \geq 2$. Conversely if $X$ is a rational normal scroll of degree $f$ containing a canonical curve, then the ruling on $X$ cuts out a pencil of divisors $\left\{D_{\lambda}\right\} \subset|D|$ such that $h^{0}\left(C, \omega_{C} \otimes \mathscr{O}_{C}(D)^{-1}\right)=f$.

Example 1.2. We consider a nonhyperelliptic canonically embedded curve $C \subset \mathbb{P}^{3}$ of genus 4 . The curve $C$ is a complete intersection of a quadric surface $Q$ and a cubic surface $S$. If $C$ admits exactly two pencils of degree 3 (which is also the maximal number), then the quadric $Q$ is isomorphic to $\mathbb{P}^{1} \times \mathbb{P}^{1}$. By Bézout's theorem, the two rulings of lines on $Q$ cut out the two pencils of degree 3 on $C$, and conversely, the quadric is the scroll of type $(1,1)$ swept out by either of these pencils. If $C$ admits only one pencil of degree 3 , then the quadric $Q$ is isomorphic to a cone (i.e., a quadric of rank 3 ) and coincides with the scroll of type $(0,2)$ swept out by the unique pencil.

In [Harris 1981] it is shown that the variety $X$ defined above is a nondegenerate $d$-dimensional variety of minimal degree $\operatorname{deg} X=f=r-d+1=\operatorname{codim} X+1$. If $e_{1}, \ldots, e_{d}>0$, then

$$
j: \mathbb{P}(\mathscr{E}) \rightarrow X \subset \mathbb{P} H^{0}\left(\mathbb{P}(\mathscr{E}), \mathscr{O}_{\mathbb{P}(\mathscr{E})}(1)\right)=\mathbb{P}^{r}
$$

is an isomorphism. Otherwise, it is a resolution of singularities. Since $R^{i} j_{*} \mathscr{O}_{\mathbb{P}(\mathscr{E})}=0$ for $i>0$, it is convenient to consider $\mathbb{P}(\mathscr{E})$ instead of $X$ for cohomological considerations.

It is known (see, e.g., [Eisenbud and Harris 1987b]) that the Picard group $\operatorname{Pic}(\mathbb{P}(\mathscr{E}))$ is generated by the class of the ruling $R=\left[\pi^{*} \mathscr{O}_{\mathbb{P} 1}(1)\right]$ and the hyperplane class $H=\left[j^{*} \mathscr{O}_{\mathbb{P r}}(1)\right]$ with intersection products

$$
H^{d}=f, \quad H^{d-1} \cdot R=1, \quad R^{2}=0 .
$$

Hence, we will write a line bundle $\mathscr{O}_{\mathbb{P}(\mathscr{E})}(a H+b R)$ in the form

$$
\mathscr{O}_{\mathbb{P}(\mathscr{E})}(a H+b R)=\pi^{*}\left(\mathscr{O}_{\mathbb{P}^{1}}(b)\right)(a H) .
$$

Theorem 1.3 [Schreyer 1986, Corollary 4.4]. Let C be a curve with a complete base point free $g_{k}^{1}$, and let $\mathbb{P}(\mathscr{E})$ be the projective bundle associated to the scroll $X$ swept out by the $g_{k}^{1}$.

(a) $C \subset \mathbb{P}(\mathscr{E})$ has a resolution $F$. of type

$$
0 \rightarrow \pi^{*} N_{k-2}(-k H) \rightarrow \pi^{*} N_{k-3}((-k+2) H) \rightarrow \cdots \rightarrow \pi^{*} N_{1}(-2 H) \rightarrow \mathscr{O}_{\mathbb{P}(\mathscr{E})} \rightarrow \mathscr{O}_{C} \rightarrow 0
$$


with

$$
N_{i}=\bigoplus_{j=1}^{\beta_{i}} \mathscr{O}_{\mathbb{P}^{1}}\left(a_{j}^{(i)}\right) \quad \text { and } \quad \beta_{i}=\frac{i(k-2-i)}{k-1}\left(\begin{array}{c}
k \\
i+1
\end{array}\right) \text {. }
$$

(b) The complex $F_{\text {. }}$ is self-dual, i.e., $\mathscr{H} o m\left(F_{\bullet}, \mathscr{O}_{\mathbb{P}(\mathscr{E})}(-k H+(f-2) R)\right) \cong F_{\text {. }}$

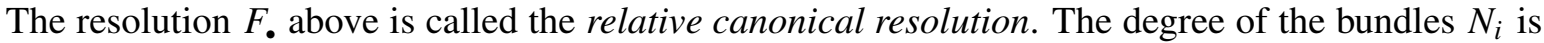
known.

Proposition 1.4 [Bopp and Hoff 2015, Proposition 2.9]. The degree of the bundle $N_{i}$ of rank $\beta_{i}=$ $\frac{k}{i+1}(k-2-i)\left(\begin{array}{c}k-2 \\ i-1\end{array}\right)$ in the relative canonical resolution $F . i s$

$$
\operatorname{deg}\left(N_{i}\right)=\sum_{j=1}^{\beta_{i}} a_{j}^{(i)}=(g-k-1)(k-2-i)\left(\begin{array}{c}
k-2 \\
i-1
\end{array}\right) .
$$

Since the rank and degree of the syzygy bundles $N_{i}$ over $\mathbb{P}^{1}$ are known, the main object of investigation is the splitting type.

Remark 1.5. Casnati and Ekedahl [1996] generalized the relative canonical resolution to finite Gorenstein covers $\pi: X \rightarrow Y$ of degree $k$. They define a relative resolution of $X \subset \mathbb{P}\left(\mathscr{E}_{T}\right)$, where $\mathscr{E}_{T}$ is the Tschirnhausen bundle on $Y$ defined by the short exact sequence

$$
0 \rightarrow \mathscr{O}_{Y} \rightarrow \pi_{*}\left(\mathscr{O}_{X}\right) \rightarrow \mathscr{E}_{T}^{\vee} \rightarrow 0
$$

Note that for a cover $C \stackrel{k: 1}{\longrightarrow} \mathbb{P}^{1}, \mathscr{E}_{T}=\mathscr{E} \otimes \mathscr{O}_{\mathbb{P}^{1}}(2)$, where $\mathscr{E}$ is the bundle associated to $\left(C, g_{k}^{1}\right)$ as in Theorem 1.3. The twists and hence the splitting types of the syzygy bundles in a resolution of $C \subset \mathbb{P}\left(\mathscr{E}_{T}\right)$ also differ from the ones in the relative canonical resolution of $C \subset \mathbb{P}(\mathscr{E})$. Indeed, following the proof of [Casnati and Ekedahl 1996, Step B, p. 445], for each $i$, the twist in the $i$-th syzygy bundle in a resolution of $C \subset \mathbb{P}\left(\mathscr{E}_{T}\right)$ differs by exactly $2 \cdot(i+1)$ from the ones given in our definition. Hence, we can deduce the degrees of the bundles in this relative resolution of $C \subset \mathbb{P}\left(\mathscr{E}_{T}\right)$ from Proposition 1.4. These degrees have also been computed directly in [Deopurkar and Patel 2018].

Definition 1.6. We say that a bundle on $\mathbb{P}^{1}$ of the form $N=\bigoplus_{j=1}^{\beta} \mathscr{O}_{\mathbb{P}^{1}}\left(a_{j}\right)$ is balanced if

$$
\max _{i, j}\left|a_{j}-a_{i}\right| \leq 1
$$

Equivalently, the bundle $N$ is balanced if $h^{1}\left(\mathbb{P}^{1}, \mathscr{E n d}(N)\right)=0$. The relative canonical resolution is called balanced if all bundles $N_{i}$ occurring in the resolution are balanced.

Remark 1.7. The locus of curves inside $\mathscr{H}_{g, k}$ that have a balanced relative canonical resolution forms an open subset of $\mathscr{H}_{g, k}$ which might be empty. Hence, to show that a generic relative canonical resolution is balanced for fixed values $(g, k)$ it is sufficient to examine a single balanced example.

Remark 1.8. The scroll associated to a general element in $\mathscr{H}_{g, k}$ is always balanced by [Ballico 1989] and [Harris 1981]. The sublocus inside $\mathscr{H}_{g, k}$ parametrizing covers such that the associated scroll is 
unbalanced defines a divisor on $\mathscr{H}_{g, k}$ precisely if $g$ is a multiple of $(k-1)$. This divisor is called the Maroni divisor (for more details on the Maroni divisor see, e.g., [van der Geer and Kouvidakis 2017] and [Deopurkar and Patel 2015]).

On the other hand, knowing the splitting type of the syzygy bundles in the relative canonical resolution for generic elements in $\mathscr{H}_{g, k}$ one can study the sublocus inside $\mathscr{H}_{g, k}$ consisting set-theoretically of curves for which a certain syzygy bundle has nongeneric splitting type. This yields interesting subvarieties which also turn out to be divisors in some cases (see [Deopurkar and Patel 2018]). Similar to Koszul divisors on the moduli space $\mathscr{M}_{g}$, the study of the divisors obtained from the relative canonical resolution sheds light on the global geometry of the Hurwitz space.

2. MaCAulay 2 PaCKage. The Macaulay 2 package [RelativeCanonicalResolution] includes various useful functions to do experiments with $k$-gonal canonical curves and the relative canonical resolution of those curves. We will briefly explain how functions in this package construct $g$-nodal $k$-gonal canonical curves of genus $g$.

The main idea is that we start with a rational normalization of the desired curve and a degree $k$ map from the normalization to $\mathbb{P}^{1}$. In the next step we choose $g$ pairs of points $\left\{P_{i}, Q_{i}\right\}$ for $i=1, \ldots, g$ on the normalization, and we glue the points in each pair to each other. If $\mathscr{L}$ is a line bundle of degree $k$ on a $g$-nodal curve $C$ with rational normalization $v: \mathbb{P}^{1} \rightarrow C$, then $\mathscr{L}$ is given as $v(\mathscr{L}) \cong \mathscr{O}_{\mathbb{P} 1}(k)$ together with gluing data between the residue class fields

$$
\frac{a_{i}}{b_{i}}: \mathscr{O}_{\mathbb{P}^{1}}(k) \otimes \mathbb{k}\left(P_{i}\right) \rightarrow \mathscr{O}_{\mathbb{P} 1}(k) \otimes \mathbb{k}\left(Q_{i}\right), i=1, \ldots, g .
$$

Let $S=\mathbb{k}[s, t]$ be the coordinate ring of $\mathbb{P}^{1}$. We start over by choosing two forms $f, h \in S_{k}$ of degree $k$ and $g$ points $R_{i}=\left(R_{i}^{(0)}: R_{i}^{(1)}\right) \in \mathbb{P}^{1}$ such that for all $i=1, \ldots, g$ the determinant

$$
\operatorname{det}\left(\begin{array}{ll}
f & R_{i}^{(0)} \\
h & R_{i}^{(1)}
\end{array}\right)=l_{i}^{0} \cdot l_{i}^{(1)} \cdot r_{i}
$$

has at least two linear factors $l_{i}^{(0)}$ and $l_{i}^{(1)}$. Note that this step might be hard to perform over a field $\mathbb{k}$ of characteristic 0 and we therefore work over a finite field. We compute $2 g$ points $P_{i}=V\left(l_{i}^{(0)}\right)$ and $Q_{i}=V\left(l_{i}^{(1)}\right)$ as the vanishing loci of these linear forms. We want to define multipliers $\left\{a_{i}, b_{i}\right\}_{i=1, \ldots, g}$ such that

$$
b_{i} \cdot f\left(P_{i}\right)=a_{i} \cdot h\left(Q_{i}\right) \text { and } b_{i} \cdot h\left(P_{i}\right)=a_{i} \cdot f\left(Q_{i}\right) \text { for } i=1, \ldots, g .
$$

By construction, we can choose $\left\{a_{i}, b_{i}\right\}_{i=1, \ldots, g}$ to be $b_{i}=1$ and $a_{i}=\frac{f\left(P_{i}\right)}{f\left(Q_{i}\right)}=\frac{h\left(P_{i}\right)}{h\left(Q_{i}\right)}$. If we define

$$
q_{i}:=\operatorname{det}\left(\begin{array}{ll}
s & P_{i}^{(0)} \\
t & P_{i}^{(1)}
\end{array}\right) \cdot \operatorname{det}\left(\begin{array}{ll}
s & Q_{i}^{(0)} \\
t & Q_{i}^{(1)}
\end{array}\right) \text { for } i=1, \ldots, g
$$


then a basis of $H^{0}\left(C, \omega_{C}\right)$ is given by

$$
\left\{s_{j}:=\prod_{i=1, i \neq j}^{g} q_{i}\right\}_{j=1, \ldots, g} .
$$

This basis $\left\{s_{j}\right\}_{j=1, \ldots, g}$ can furthermore be modified in such a way that the scroll defined by the line bundle of degree $k$ will have a "normalized" form, i.e., the $2 \times(g-k+1)$ matrix defining the scroll will consist of blocks of the form

$$
\left(\begin{array}{cc}
t_{i} & t_{i+2} \\
t_{i+1} & t_{i+3}
\end{array}\right)
$$

where $T=\mathbb{k}\left[t_{0}, \ldots, t_{g-1}\right]$ is the coordinate ring of $\mathbb{P}^{g-1}$.

In the package [RelativeCanonicalResolution] we also provide a function that describes the generators of the ideal of $C$ in terms of elements of the Cox ring of the scroll $\mathbb{P}(\mathscr{E})$.

Remark 2.1. There is an explicit identification

$$
H^{0}\left(\mathbb{P}(\mathscr{E}), \mathscr{O}_{\mathbb{P}(\mathscr{E})}(a H+b R)\right) \cong H^{0}\left(\mathbb{P}^{1},\left(S_{a} \mathscr{E}\right)(b)\right) \text { for } a \geq 0,
$$

where $S_{a} \mathscr{E}$ is the $a$-th symmetric power of the vector bundle $\mathscr{E}$ (see [Schreyer 1986, (1.3)]). This gives a description of the coordinate ring

$$
R_{\mathbb{P}(\mathscr{E})}=\bigoplus_{a, b \in \mathbb{Z}} H^{0}\left(\mathbb{P}(\mathscr{E}), \mathscr{O}_{\mathbb{P}(\mathscr{E})}(a H+b R)\right)
$$

of $\mathbb{P}(\mathscr{E})$ as the Cox ring $\mathbb{k}\left[v, w, \varphi_{0}, \ldots, \varphi_{d-1}\right]$ equipped with bigrading deg $v=\operatorname{deg} w=(1,0)$ and $\operatorname{deg} \varphi_{i}=\left(e_{1}-e_{i+1}, 1\right)$.

Finally the relative canonical resolution of $C \subset \mathbb{P}(\mathscr{E})$ can be computed by successively picking syzygies in correct degrees.

Example 2.2. We compute a nodal 6-gonal canonical curve of genus 9 .

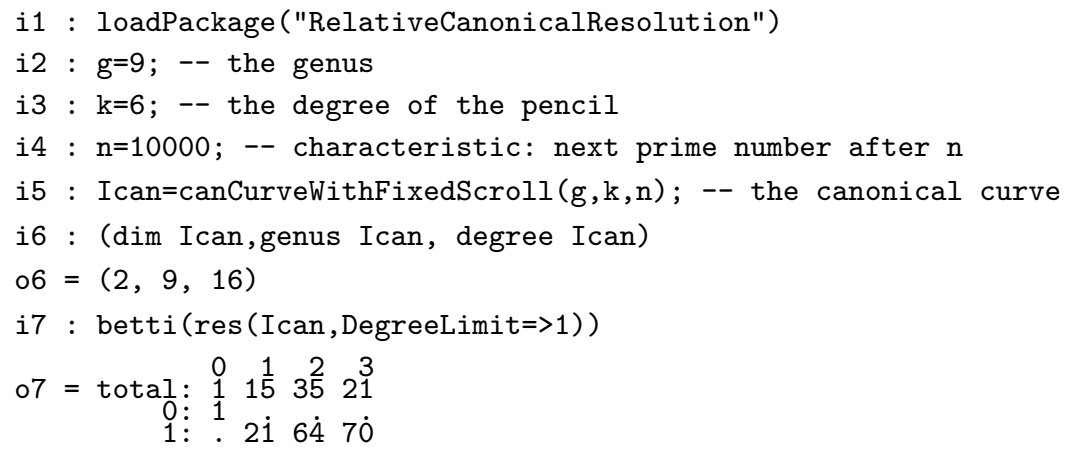

Next we compute the ideal of $C$ inside the Cox ring of the scroll $\mathbb{P}(\mathscr{E})$.

$$
\begin{aligned}
\text { i8 : Jcan=curveOnScroll }(\text { Ican, }, \mathrm{k}) \text {; -- the curve inside the scroll } \\
\text { i9: RX=ring Jcan; -- the bigraded Cox ring of the scroll } \\
\text { o9 = } \\
\quad-\frac{\mathrm{ZZ}}{10007}\left[\mathrm{pp}_{0}, \mathrm{pp}_{1}, \mathrm{pp}_{2}, \mathrm{pp}_{3}, \mathrm{pp}_{4}, \mathrm{v}, \mathrm{w}\right]
\end{aligned}
$$


We compute the relative canonical resolution:

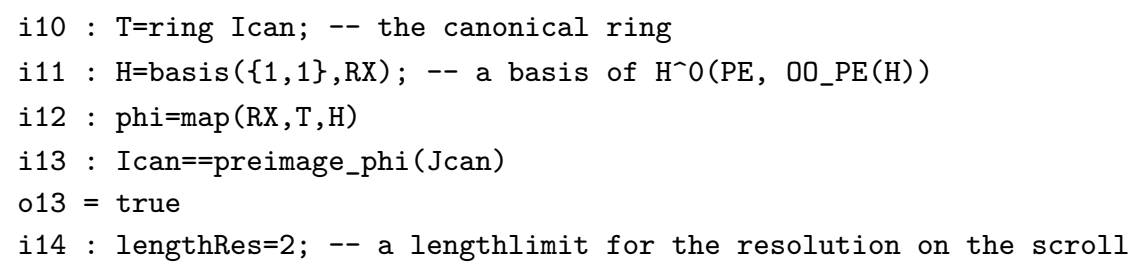

With respect to the total degree, the Betti table of the relative canonical resolution has the following form:

-- the relative canonical resolution:

i15 : betti (resX=resCurveOnScroll (Jcan,g, lengthRes))

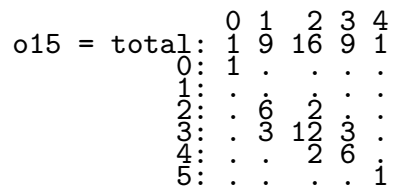

The scroll cut out by the $g_{6}^{1}$ on $C$ has the following normalized determinantal representation:

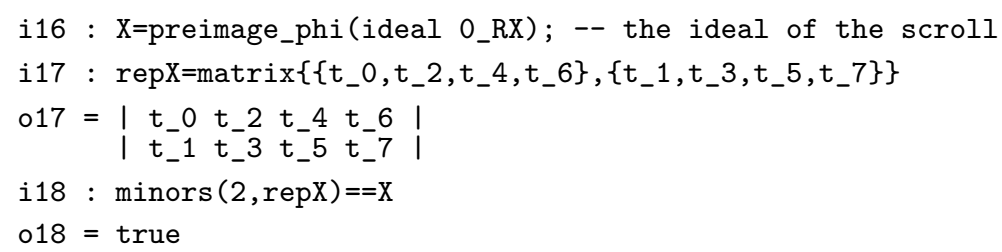

Remark 2.3. By o15, we see that the second syzygy bundle $N_{2}$ is unbalanced in our example. Although this single example does not show that the generic relative canonical resolution is unbalanced for this case, one can show that this is indeed the generic form (see [Bopp and Hoff 2017]).

\section{EXPERIMENTS AND CONJECTURES.}

Database of experiments. Using our Macaulay2 package [RelativeCanonicalResolution] we have computed the relative canonical resolution for various cases. For nonhyperelliptic, generic curves of genus $g \leq 23$ with a pencil of degree $3 \leq k \leq \min \{g-1,14\}$, all expected Betti tables are listed on the webpage [Blug et al. 2018].

The web page was set up with the help of Sascha Blug. All the experiments that led to Betti tables in [Blug et al. 2018] were performed over a finite field. If the examples for certain values $(g, k)$ yield a balanced relative canonical resolution, then by semi-continuity one can conclude that this is indeed the general behavior (even for complex algebraic curves).

Since changing the characteristic for the unbalanced cases did not change the shape of the Betti tables, we believe that the Betti tables in [Blug et al. 2018] reflect the generic behavior. In general, we do not have a proof of this statement. However, it has been determined in some cases whether the first bundle $N_{1}$ is balanced (see [Bopp and Hoff 2015] and [Bujokas and Patel 2015]). For several cases our examples 
lead to a conjecture that certain higher syzygy bundles in the relative canonical resolution are unbalanced. Most of these cases remain mysterious.

Syzygy divisors on Hurwitz spaces. Deopurkar and Patel used the relative canonical resolution to describe new effective divisors on the Hurwitz scheme $\mathscr{H}_{g, k}$. If the degree $k$ divides $g-1$, it is shown in [Bujokas and Patel 2015] that the relative canonical resolution for a generic element in $\mathscr{H}_{g, k}$ is totally balanced, and hence, the locus $\mu_{i}$, corresponding set-theoretically to covers in $\mathscr{H}_{g, k}$ for which the $i$-th syzygy bundle $N_{i}$ is unbalanced, has expected codimension 1. Deopurkar and Patel [2018] give these syzygy divisors $\mu_{1}, \ldots, \mu_{k-3}$ a scheme structure and compute their classes in a partial compactification of the Hurwitz scheme $\widetilde{\mathscr{H}}_{g, k}$. In their main theorem, they represent the divisor classes $\left[\mu_{i}\right]$ in terms of certain tautological classes $\kappa, \zeta$ and $\delta$ (see [Deopurkar and Patel 2018, §2] for the precise definition of those classes).

Theorem 3.1 [Deopurkar and Patel 2018, Theorem 1.1]. Suppose $k$ divides $g-1$. Let $i$ be an integer with $1 \leq i \leq k-3$. The locus $\mu_{i} \subset \widetilde{\mathscr{H}}_{g, k}$ is an effective divisor whose class in $\mathrm{Pic}_{\mathbb{Q}}\left(\widetilde{\mathscr{H}}_{g, k}\right)$ is given by

$$
\left[\mu_{i}\right]=A_{i} \cdot\left(6(g k-6 g+k+6) \cdot \zeta-k(k-12) \cdot \kappa-k^{2} \cdot \delta\right),
$$

where

$$
A_{i}:=\frac{(k-2)(k-3)}{6(i+1)(k-i-1)} \cdot\left(\begin{array}{c}
k-4 \\
i-1
\end{array}\right)^{2} .
$$

Note that all the classes $\left[\mu_{i}\right]$ are proportional. The same phenomenon appears for classes of divisorial Brill-Noether loci in the moduli space $\overline{\mathscr{M}}_{g}$ (see [Eisenbud and Harris 1987a] and [Harris and Mumford 1982]). For the divisorial Brill-Noether classes it is known that these classes are supported on different sets, and in [Deopurkar and Patel 2018] the authors conjecture that this also happens for the syzygy divisors on $\mathscr{H}_{g, k}$.

We come to a different conclusion. Computing various examples of curves and their relative canonical resolution for $(g, k) \in\{(6,13),(7,15),(8,17),(6,19)\}$ over a field of small characteristic $p \leq 500$ we found the following pattern which we conjecture to be true in general. Note that the probability of a random computed example to end up in a certain codimension 1 locus is roughly $\frac{1}{p}$.

Conjecture 1. Let $n$ and $k$ be integers, and $g-1=n \cdot k$. Let $i$ be an integer with $1 \leq i \leq k-3$. For $a$ general element $\left(C, g_{k}^{1}\right) \in \mu_{i} \subset \widetilde{\mathscr{H}}_{g, k}$, let $N_{j}$ be the $j$-th syzygy bundle in the relative canonical resolution of $C$ with $1 \leq j \leq k-3$. Then $N_{j}$ is unbalanced and the splitting type of $N_{j}$ is

$$
N_{j}=\mathscr{O}_{\mathbb{P} 1}((n-1)(j+1)-1)^{\oplus\left(\begin{array}{c}
k-4 \\
j-1
\end{array}\right)} \oplus \mathscr{O}_{\mathbb{P} 1}((n-1)(j+1))^{\mathrm{rk} N_{j}-2 \cdot\left(\begin{array}{c}
k-4 \\
j-1
\end{array}\right)} \oplus \mathscr{O}_{\mathbb{P} 1}((n-1)(j+1)+1)^{\oplus\left(\begin{array}{c}
k-4 \\
j-1
\end{array}\right)} .
$$

In particular, all the effective divisors $\mu_{i}$ are supported on the same set.

Remark 3.2. One can easily check that the number $A_{i}$ in Theorem 3.1 is precisely

$$
A_{i}=\frac{1}{6 k} \cdot \mathrm{rk} N_{i} \cdot\left(\begin{array}{c}
k-4 \\
i-1
\end{array}\right) \text {. }
$$


Conjecture 1 predicts that the factor $\left(\begin{array}{c}k-4 \\ i-1\end{array}\right)$ of $A_{i}$ also measures how unbalanced the bundle $N_{i}$ is.

Remark 3.3. If $(g-1) \neq n \cdot k$ then one can still consider the jumping loci set-theoretically defined as the subset of $\widetilde{\mathscr{H}}_{g, k}$ consisting of covers such that the $i$-th syzygy bundle in the relative canonical resolution does not have generic splitting type. As in the divisorial case, one could ask if all those loci are supported on the same set. Experiments using our package [RelativeCanonicalResolution] show that there are several examples where these jumping loci have different support.

Further conjectures. We state several conjectures concerning the shape of relative canonical resolutions. This has partly also been discussed in [Bopp and Hoff 2015]. We refer to [Arbarello et al. 1985] for basics about Brill-Noether theory. Recall that the Brill-Noether number is defined as $\rho(g, k, r):=$ $g-(r+1)(g-k+r)$ for integers $g, k$ and $r$.

Conjecture 2. Let $C \subset \mathbb{P}^{g-1}$ be a general canonical curve, and let $k$ be a positive integer such that $\rho:=\rho(g, k, 1) \geq 0$, and let $g_{k}^{1}$ be a general pencil in $W_{k}^{1}(C)$. Then for bundles $N_{i}=\bigoplus \mathscr{O}_{\mathbb{P}^{1}}\left(a_{j}^{(i)}\right)$, $i=2, \ldots,\left\lceil\frac{k-3}{2}\right\rceil$, there is the bound

$$
\max _{j, l}\left|a_{j}^{(i)}-a_{l}^{(i)}\right| \leq \min \{g-k-1, i+1\} .
$$

This bound is furthermore sharp in the following sense. Given two integers $k \geq 3$ and $2 \leq i \leq\left\lceil\frac{k-3}{2}\right\rceil$, there exists an integer $g$ such that the general canonical curve $C$ of genus $g$ has an $i$-th syzygy bundle $N_{i}$ in the relative canonical resolution, associated to a general pencil in $W_{k}^{1}(C)$, that satisfies $\max _{j, l}\left|a_{j}^{(i)}-a_{l}^{(i)}\right|=$ $\min \{g-k-1, i+1\}$. In particular, if $g-k=2$, the relative canonical resolution is balanced.

Remark 3.4. Conjecture 2 in the case $g-k=2$ says that the bundles in the relative canonical resolution are of the form

$$
N_{i}=\mathscr{O}_{\mathbb{P} 1}^{\oplus i \cdot\left(\begin{array}{l}
g-4 \\
i+1
\end{array}\right)} \oplus \mathscr{O}_{\mathbb{P} 1}(1)^{\oplus(g-4-i) \cdot\left(\begin{array}{c}
g-4 \\
g-3-i
\end{array}\right)} .
$$

Note that the Betti numbers $i \cdot\left(\begin{array}{c}k-2 \\ i+1\end{array}\right)$ appearing in the conjecture are the Betti numbers of a rational normal curve of degree $k-2$.

We also verified Conjecture 3 for $g \leq 23$.

Conjecture 3. For a general cover $C \rightarrow \mathbb{P}^{1}$ in $\mathscr{H}_{g, k}$ with $\rho(g, k, 1) \leq 0$, the bundle $N_{1}$ is balanced.

ACKNOWLEDGEMENTS. We would like to thank Sascha Blug for setting up the web page [Blug et al. 2018] that displays all the experimental data. This work is a contribution to the Project 1.7 of the SFBTRR 195 "Symbolic Tools in Mathematics and their Application" of the German Research Foundation (DFG). We also thank the referees for suggestions and comments improving the presentation of the article and the Macaulay2 package.

SUPPLEMENT. The online supplement contains version 1.0 of [RelativeCanonicalResolution]. 


\section{REFERENCES.}

[Arbarello et al. 1985] E. Arbarello, M. Cornalba, P. A. Griffiths, and J. Harris, Geometry of algebraic curves, I, Grundlehren der Mathematischen Wissenschaften [Fundamental Principles of Mathematical Sciences] 267, Springer, 1985. MR

[Ballico 1989] E. Ballico, "A remark on linear series on general k-gonal curves”, Boll. Un. Mat. Ital. A (7) 3:2 (1989), $195-197$. MR

[Blug et al. 2018] S. Blug, C. Bopp, and M. Hoff, "Relative canonical resolutions", 2018, available at https://www.math.uni-sb.de/ ag/schreyer/images/data/computeralgebra/relcanres/html/.

[Bopp and Hoff 2015] C. Bopp and M. Hoff, "Resolutions of general canonical curves on rational normal scrolls", Arch. Math. (Basel) 105:3 (2015), 239-249. MR

[Bopp and Hoff 2017] C. Bopp and M. Hoff, "Moduli of lattice polarized K3 surfaces via relative canonical resolutions", preprint, 2017. arXiv

[Bujokas and Patel 2015] G. Bujokas and A. Patel, "Invariants of a general branched cover of $\mathbb{P}^{1}$ ”, preprint, 2015. arXiv

[Casnati and Ekedahl 1996] G. Casnati and T. Ekedahl, "Covers of algebraic varieties, I: A general structure theorem, covers of degree 3, 4 and Enriques surfaces”, J. Algebraic Geom. 5:3 (1996), 439-460. MR

[Deopurkar and Patel 2015] A. Deopurkar and A. Patel, "The Picard rank conjecture for the Hurwitz spaces of degree up to five", Algebra Number Theory 9:2 (2015), 459-492. MR

[Deopurkar and Patel 2018] A. Deopurkar and A. Patel, "Syzygy divisors on Hurwitz spaces", pp. 209-222 in Higher genus curves in mathematical physics and arithmetic geometry, edited by A. Malmendier and T. Shaska, Contemp. Math. 703, Amer. Math. Soc., Providence, RI, 2018. MR

[Eisenbud and Harris 1987a] D. Eisenbud and J. Harris, "The Kodaira dimension of the moduli space of curves of genus $\geq 23$ ", Invent. Math. 90:2 (1987), 359-387. MR

[Eisenbud and Harris 1987b] D. Eisenbud and J. Harris, "On varieties of minimal degree (a centennial account)", pp. 3-13 in Algebraic geometry (Brunswick, Maine, 1985), edited by S. J. Bloch, Proc. Sympos. Pure Math. 46, Amer. Math. Soc., Providence, RI, 1987. MR

[Farkas 2009] G. Farkas, "Koszul divisors on moduli spaces of curves", Amer. J. Math. 131:3 (2009), 819-867. MR

[Farkas 2018] G. Farkas, "Effective divisors on Hurwitz spaces", preprint, 2018. arXiv

[van der Geer and Kouvidakis 2017] G. van der Geer and A. Kouvidakis, "The cycle classes of divisorial Maroni loci", Int. Math. Res. Not. 2017:11 (2017), 3463-3509. MR

[Harris 1981] J. Harris, "A bound on the geometric genus of projective varieties", Ann. Scuola Norm. Sup. Pisa Cl. Sci. (4) 8:1 (1981), 35-68. MR

[Harris and Mumford 1982] J. Harris and D. Mumford, "On the Kodaira dimension of the moduli space of curves", Invent. Math. 67:1 (1982), 23-88. MR

[Hirschowitz and Ramanan 1998] A. Hirschowitz and S. Ramanan, "New evidence for Green's conjecture on syzygies of canonical curves", Ann. Sci. École Norm. Sup. (4) 31:2 (1998), 145-152. MR

[Macaulay2] D. R. Grayson and M. E. Stillman, "Macaulay2: a software system for research in algebraic geometry", available at http://www.math.uiuc.edu/Macaulay2.

[RelativeCanonicalResolution] C. Bopp and M. Hoff, "RelativeCanonicalResolution - construction of relative canonical resolutions and Eagon-Northcott type complexes", Macaulay2 package, available at https://www.math.uni-sb.de/ag/schreyer/ index.php/computeralgebra.

[Schreyer 1986] F.-O. Schreyer, "Syzygies of canonical curves and special linear series", Math. Ann. 275:1 (1986), 105-137. MR

[Voisin 2005] C. Voisin, “Green's canonical syzygy conjecture for generic curves of odd genus”, Compos. Math. 141:5 (2005), 1163-1190. MR 


\section{CHRISTIAN BOPP:}

bopp@math.uni-sb.de

Universität des Saarlandes, Saarbrücken, Germany

Michael HofF:

hahn@math.uni-sb.de

Universität des Saarlandes, Saarbrücken, Germany 

Phylogenetic trees

Hector Baños, Nathaniel Bushek, Ruth Davidson, Elizabeth Gross, Pamela E.

Harris, Robert Krone, Colby Long, Allen Stewart and Robert Walker

Software for doing computations in graded Lie algebras

Clas Löfwall and Samuel Lundqvist

The relative canonical resolution: Macaulay2-package, experiments and conjectures

Christian Bopp and Michael Hoff

The FrobeniusThresholds package for Macaulay2

Daniel J. Hernández, Karl Schwede, Pedro Teixeira and Emily E. Witt

Computing theta functions with Julia

Daniele Agostini and Lynn Chua

Decomposable sparse polynomial systems

Taylor Brysiewicz, Jose Israel Rodriguez, Frank Sottile and Thomas Yahl

A package for computations with sparse resultants

Giovanni Staglianò

ExteriorModules: a package for computing monomial modules over an exterior algebra

Luca Amata and Marilena Crupi

The Schur-Veronese package in Macaulay2

Juliette Bruce, Daniel Erman, Steve Goldstein and Jay Yang

admcycles - a Sage package for calculations in the tautological ring of the moduli space of stable curves

Vincent Delecroix, Johannes Schmitt and Jason van Zelm

Coding theory package for Macaulay2

Taylor Ball, Eduardo Camps, Henry Chimal-Dzul, Delio Jaramillo-Velez, Hiram

López, Nathan Nichols, Matthew Perkins, Ivan Soprunov, German Vera-Martínez and Gwyn Whieldon

Threaded Gröbner bases: a Macaulay2 package

Sonja Petrović and Shahrzad Zelenberg

Standard pairs of monomial ideals over nonnormal affine semigroups in SageMath Byeongsu Yu

Computations with rational maps between multi-projective varieties Giovanni Staglianò 